\title{
X-Ray Standing Waves at Crystal Surfaces
}

\section{Citation}

Cowan, P. L., J. A. Golovchenko, and M. F. Robbins. 1980. X-Ray Standing Waves at Crystal

Surfaces. Physical Review Letters 44, no. 25: 1680-1683. doi:10.1103/physrevlett.44.1680.

\section{Published Version}

doi:10.1103/PhysRevLett.44.1680

\section{Permanent link}

http://nrs.harvard.edu/urn-3:HUL.InstRepos:29407057

\section{Terms of Use}

This article was downloaded from Harvard University's DASH repository, and is made available under the terms and conditions applicable to Other Posted Material, as set forth at http:// nrs.harvard.edu/urn-3:HUL.InstRepos:dash.current.terms-of-use\#LAA

\section{Share Your Story}

The Harvard community has made this article openly available.

Please share how this access benefits you. Submit a story.

\section{Accessibility}




\title{
X-Ray Standing Waves at Crystal Surfaces
}

\author{
P. L. Cowan, (a) J. A. Golovchenko, and M. F. Robbins \\ Bell Laboratories, Murvay Hill, New Jersey 07974 \\ (Received 17 March 1980)
}

\begin{abstract}
With use of bromine atoms, deposited on the surface of a silicon single crystal, the presence of $x$-ray standing waves above the crystal surface has been detected under conditions of strong Bragg diffraction.
\end{abstract}

PACS numbers: 68.20.+t, 61.10.Fr

Nearly ninety years ago Wiener ${ }^{1}$ reported on a method of exciting standing waves of optical intensity just outside the surface of a metallic reflecting mirror. In so doing he determined the correspondence between the electric vector of Maxwell's theory and the photographically active light vector which developed a plate placed just outside the mirror surface. We report below on an extension of Wiener's idea to the $\mathrm{x}$-ray region of the spectrum.

Our interest in this problem lies not only in demonstrating phenomena analogous to that observed by Wiener but also in the prospect of obtaining a completely new experimental method to observe and characterize, on a microscopic scale, adsorbed layers on the surfaces of single crystals. We describe the experimental realization of these possibilities in the following discussion.

The basic idea behind our experiment is quite similar to that of Wiener's, i.e., establish a standing-wave field external to the crystal surface via the interference between an incident and reflected plane wave. In place of metallic reflection we have used a Bragg reflection for the $\mathrm{x}$-ray case. This distinction results in a remarkable extension of the achievable experimental possibilities compared to metallic reflection. In the latter case the phase of the reflected wave relative to the incident wave is determined by the fixed boundary condition of zero tangential electric vector along the surface. This fixes the phase of the standing wave relative to the boundary surface. The conditions that determine the phase of the reflected beam from a Bragg reflection are somewhat more complex but are thoroughly exposed in the dynamical theory of $x$-ray diffraction. Thus as early as 1935 , von Laue $^{2}$ discussed the nature of the $\mathrm{x}$-ray standing waves excited inside perfect crystals for both the Bragg and Laue diffraction geometries. The Bragg case is particularly interesting to us. Here for semiinfinite single crystals a finite angular region of essentially total reflection occurs in the vicinity of the Bragg angle. Within this region the phase of the reflected beam is a very strong function of angle, changing by $\pi$ from one side of the totalreflection region to the other. Thus the resulting standing wave caused by interference between incident and reflected beams is no longer fixed relative to the surface but may be moved experimentally with a simple angular adjustment. A second distinction arising from the use of a Bragg rather than a metallic reflection lies in the configuration of the standing-wave field. The Bragg condition implies that the nodal planes of the standing waves lie parallel to, and have the periodicity of, the Miller planes responsible for the diffraction.

The final and crucial aspect of our experiment is to replace the photographic emulsion of Wiener's experiment with an atomic layer of surface impurity atoms and detect the presence of $x$-ray standing waves in their vicinity through $\mathrm{x}$-ray fluorescent scattering. Thus as the incident beam angle is scanned through the region of total reflectivity, the standing waves outside the crystal surface will move, and as a node (or antinode) passes through the atomic position of the surface atoms the characteristic fluorescent impurity signal should go through a minimum (or maximum). This experiment is a logical extension of recent work developing $x$-ray standing-wave methods for the determination of bulk atomic impurity locations. ${ }^{3}$

The conventional approach to the preparation of a crystal surface with adatoms involves sputtering, annealing, and impurity-atom exposure followed by a number of standard measurements such as low-energy electron diffraction (LEED), Auger spectroscopy, etc., all done in an ultrahigh-vacuum environment. While we have pursued this route in some of our surface $x$-ray interference experiments, we report on a different kind of surface preparation here. We have taken advantage of the strong penetrating power of both incident and fluorescence $x$ rays to demonstrate the existence of surface standing waves 
at a crystal-liquid boundary. We believe that this represents one of the few means of obtaining microscopic atomic location information at this kind of interface.

A single crystal of silicon was cut and Sytonpolished with the (220) planes parallel to the surface. Oxide was removed with an $\mathrm{HF}$ etch followed by a quick methanol rinse. The sample was then immediately transferred to a solution of 50 $\mathrm{ml}$ methyl alcohol to which a single drop of liquid bromine was added, the goal being to deposit bromine atoms on the clean silicon surface. Without allowing the sample surface to come in contact with the atmosphere it was transferred successively to six clean methanol baths. A 0.0005in. Mylar window wet with methanol was placed in contact with the sample and the combination of surface wetting and tension forces trapped a thin liquid layer (several microns thick). The sample was then placed in a quartz cell (see Fig. 1) that continually resupplied methanol to the layer via capillary action replacing liquid lost by evaporation from the sample edges. This procedure is necessary to minimize Compton and diffuse scatterings off the liquid from entering our fluorescence detector.

The cell described above was then placed in an $\mathrm{x}$-ray apparatus illustrated in Fig. 1. A finefocus molybdenum $x$-ray tube served as the source of Mo $K \alpha$ x rays. The beam was then collimated and monochromated by a 220 reflection from an asymmetrically cut silicon crystal. The asymmetry of the collimator crystal produced an outgoing beam having a theoretical angular width $\frac{1}{7}$ the natural width of the (220) reflection for a symmetric crystal. This broad, highly

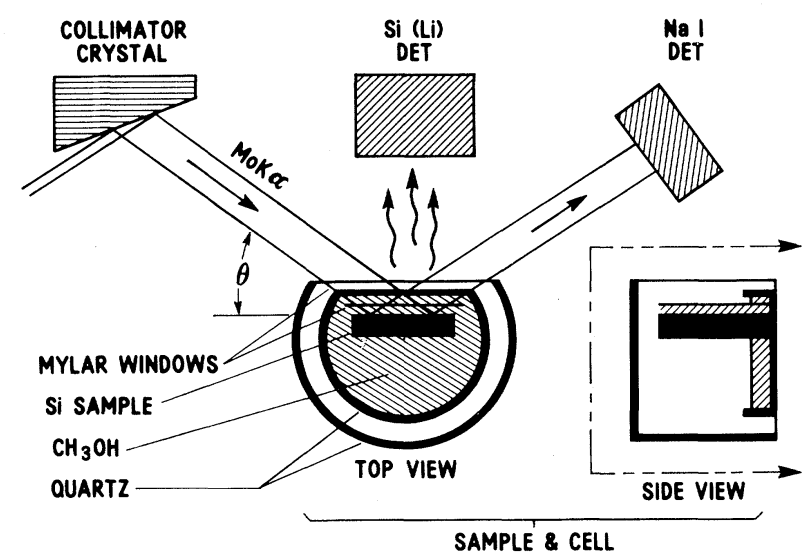

FIG. 1. Schematic layout of experimental apparatus including sample cell detail. collimated beam then fell upon our sample in solution after passing through the thin Mylar window. The sample and its holder were placed on a recently discussed ${ }^{4}$ piezoelectrically controlled and long-term stabilized goniometer capable of stabilities of order 0.01 arc sec over many days. The goniometer was programmed to sweep back and forth through the region of total reflection associated with the (220) Bragg angle (10.6 ${ }^{\circ}$. A lithium-drifted silicon $\mathrm{x}$-ray detector monitored the inelastic $x$ rays scattered from the sample. In particular the fluorescent $x$-ray yield from the surface bromine atoms was monitored as a function of crystal angle relative to the beam.

Bromine surface coverage could be evaluated immediately by comparing "off Bragg” bromine fluorescence yields with another similar sample that had $10^{15} / \mathrm{cm}^{2}$ bromine atoms ion-implanted to a depth of $\sim 300 \AA$ instead of the chemical treatment. A surface coverage of $4 \times 10^{14}$ bromine $/ \mathrm{cm}^{2}$ was thus obtained fro the chemically prepared sample. Evidence that the chemically deposited bromine was actually on the surface came from observing that a brief dip in hydrofluoric acid completely removed it. (We have also attempted to establish bromine coverage by Rutherford backscattering and electron-induced Auger yields, but bromine appears to be rapidly desorbed by these techniques.)

The closed-circle data points in Fig. 2(a) which were acquired over a 14-h period represent the experimental angular yield of bromine $K \alpha$ fluorescence around the total-reflection region. The yield is clearly asymmetric with respect to the center of the reflectivity curve which is shown as curve $A$ in the figure. Had there been no standing waves at the surface or no coherent bromine atom positions this yield would have been simply an incoherent sum of fluorescence yields from the incident and reflected beams (curve $B$ ).

We proceed now to discuss the coherent aspects of the bromine fluorescence signal. First note that as we move across the region of total reflectivity the aforementioned phase change of the reflected beam results in the $\mathrm{x}$-ray antinodes shifting from being midway between to being in step withf the crystal (220) planes. Thus, for an angle of incidence at the left of the total-reflection region the first external $x$-ray antinode lies one half a (220) planar spacing above the surface. For an angle of incidence at the right of the totalreflection region the antinode lies one whole planar spacing above the surface $(1.92 \AA)$. There- 


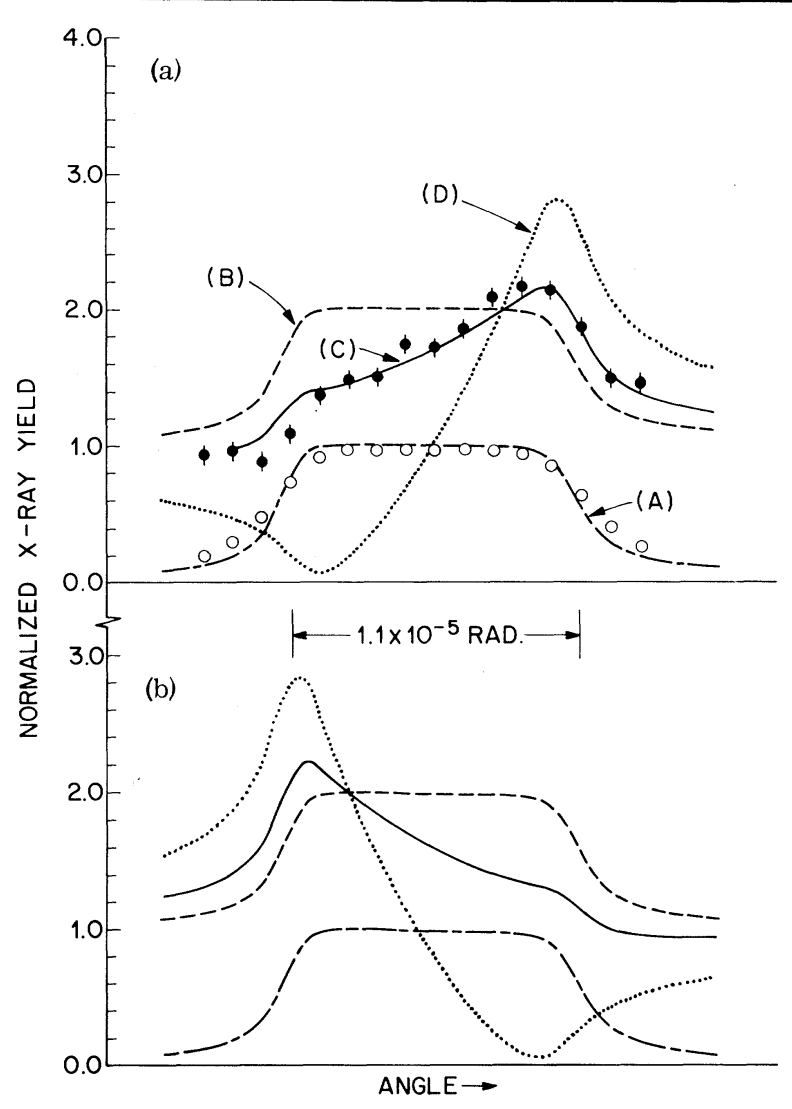

FIG. 2. (a) Normalized $x$-ray yields from both fluorescence and reflected-beam detectors. Bromine fluorescence, solid circles; Bragg reflectivity, open circles. Curve $A$, Bragg reflectivity theory; curve $\boldsymbol{B}$, standing-wave theory with coherent fraction $0 \%$; curve $C$, standing-wave theory with coherent fraction $30 \%$ at $\delta=1.72 \AA$; curve $D$, standing-wave theory with coherent fraction $100 \%$ at $\delta=1.72 \AA$. $\delta$ is distance above surface plane. (b) Bragg reflectivity theory, dot-dashed curve; standing-wave theory with coherent fraction $0 \%$, dashed curve; standing-wave theory with coherent fraction $30 \%$ at $\delta=1.15 \AA$, solid curve; standing-wave theory with coherent fraction $100 \%$ at $\delta=1.15 \AA$, dotted curve.

fore the presence of the fluorescence maximum on the right side of the total-reflectivity region indicates that a significant fraction of the adsorbed bromine lies approximately $1.9 \AA$ above the crystal surface. A calculation using standard silicon parameters in a two-beam dynamical theory is also shown in Fig. 2(a) (curve $C$ ). The fit assumes that $30 \%$ of the bromine lies $1.73 \AA$ above the crystal surface and $70 \%$ is incoherent relative to the crystal lattice below. The theory for completely coherent (curve $D$ ) and completely incoherent (curve $B$ ) results is also indicated. Figure 2(b) shows the expected results for com- pletely incoherent, completely coherent, and a mixture with a different atomic position than we have observed. Our experimental value of 1.73 $\pm 0.07 \AA$ corresponds closely with that expected from a $\mathrm{Si}-\mathrm{Br}$ covalent bond in volatile molecules. The value for the latter is ${ }^{15} 1.76 \pm 0.16 \AA$ with geometrical factors taken into account. Here we assume that the angular orientation of the $\mathrm{Si}-\mathrm{Br}$ bond is identical to that expected for the equivalent $\mathrm{Si}-\mathrm{Si}$ bond.

It has been shown previously ${ }^{3 \mathrm{~d}}$ that the incident and diffracted beams are highly coherent even at distances of $\sim 1000 \AA$ above the single-crystal region which produces the standing waves. We therefore believe that the explanation for the $65 \%$ incoherent signal is actually connected with some bromine atom sites being essentially uncorrelated with the crystal lattice below. Thus, while some of the deposited $\mathrm{Br}$ atoms are able to situate themselves at preferred sites relative to the crystal substrate, most of the Br present may be caught in some more complex structure. Surface impurities from the air of the methanol such as oxygen or hydrocarbons which are too light to give an observable fluorescence signal may be responsible for the complex environment. On the other hand, while it is not appropriate to go into great detail here, we just mention that samples prepared and measured in a UHV environment, samples allowed to age in air after the bromine treatment, and samples prepared in a $\mathrm{CF}_{3} \mathrm{Br}$ plasma all gave rather similar results with regard to bromine incoherent fraction (and position). Attempts to get any coherent fraction from bromine in the bulk (i.e., ion implantation followed by thermal or laser annealing), however, have all failed. This leaves open the possibility that some bromine may actually penetrate into the solid and take up incoherent positions or that a combination of locations is populated that simulates an incoherent result in the fluorescence data.

The statistical accuracy of the data in Fig. 2 is rather poor compared to data from standing-wave experiments on bulk impurities. ${ }^{3}$ This is due partly to the low coherent fraction $\left(\sim 10^{14} \mathrm{Br} / \mathrm{cm}^{2}\right)$ and partly to the limited times we have been able to keep a well-defined liquid-solid interface. However, both surface and bulk $x$-ray standingwave experiments could be drastically shortened in duration by use of $x$-ray synchrotron radiation as a source of the incident $x$-ray beam. In addition to the increased incident flux (by factors of $\sim 10^{3}$ ) other major advantages of such a source include reductions in the Compton and thermal 
diffuse backgrounds by utilizing the beam polarization, and optimization of fluorescence cross sections by beam energy tuning. We believe that with these improvements a position resolution better than 0.01-0.02 $\AA$ can be obtained for surface impurities in relatively short experiments.

In conclusion, we have demonstrated the existence of $\mathrm{x}$-ray standing waves outside a silicon crystal surface under condition of strong Bragg diffraction. Such fields can be excited not only near the crystal-vacuum interface but also at the crystal-liquid interface. Their use in the systematic study of surface atom location in both environments may be expected to contribute significantly to the study of surface physics in the near future.

The authors would like to acknowledge the fruitful discussions with Professor R. P. Van Duyne of Northwestern University and B. A. Parkinson of Bell Laboratories which led to the successful preparation of our samples. M. Bedzyk and K. Englis from State University of New York at Albany, and B. Hughey and D. Kaplan from Princeton University, provided assistance in performing some of the experiments. We also acknowledge encouragement and advice from W. L. Brown and A. Heller of Bell Laboratories. R. A. Levesque, R. Heckmann, and G. L. Miller of Bell Laboratories all contributed valuable technical assis- tance in developing the capabilities demonstrated in this paper.

\footnotetext{
${ }^{(a)}$ Present address: National Bureau of Standards, Washington, D. C. 20234.

${ }^{1}$ O. Wiener, Ann. Phys. Chem. $\underline{40}, 203$ (1890); for a discussion of Wiener's experiment in English see A. Sommerfeld, Optics (Academic, New York, 1954), p. $56-58$.

${ }^{2}$ M. V. Laue, Ann. Phys. (Leipzig) 23, 705 (1935).

${ }^{3 a}$ B. W. Batterman, Phys. Rev. 133, A759 (1964).

${ }^{3 b}$ B. W. Batterman, Phys. Rev. Lett. 22, 703 (1969).

${ }^{3 \mathrm{c}} \mathrm{J}$. A. Golovchenko, B. W. Batterman, and W. L. Brown, Phys. Rev. B 10, 4239 (1974).

${ }^{3 \mathrm{~d}} \mathrm{~S}$. K. Anderson, J.A. Golovchenko, and G. Mair, Phys. Rev. Lett. 37, 1141 (1976).

${ }^{3 e}$ M. V. Kruglov, V. N. Shchemeley, and G. G. Kareva, Phys. Status Solidi (a) 46, 343 (1978).

${ }^{4}$ G. L. Miller, R. Boie, P. L. Cowan, J. A. Golovchenko, R. Kerr, and D. Robinson, Rev. Sci. Instrum. 50, 8 (1979). S. K. Andersen, P. K. Bhattacharya, J. Golovchenko, N. Hertel, and G. Mair, J. Phys. E $\underline{12}, 1063$ (1979).

${ }^{5}$ Halogen Chemistry, edited by Viktor Gutmann (Academic, New York, 1967), Vol. 2. See also Tables of Interatomic Distances and Configuration in Molecules and Ions, edited by L. E. Sutton (Chemical Society, London, 1958), where considerably smaller and perhaps optimistic error limits are claimed.
} 